\title{
KOREA: WACHSTUM UND ENTWICKLUNG?
}

I.

Nächst einigen Erdölstaaten hat Südkorea in den beiden letzten Jahren die höchsten Wachstumsraten seiner Wirtschaft erzielt. Sie wurden stimuliert durch den massiven Zustrom ausländischen Kapitals, zunächst von Wirtschafts- und Militärhilfe, letztlich vornehmlich durch Privatinvestitionen und Lieferantenkredite. Natürliche Rohstoffe standen fast kaum zur Verfügung; nur der ,,Faktor Arbeitskraft“" war reichlich vorhanden, über den ja auch andere Entwicklungsländer im Überfluß verfügen. Korea hat eine der höchsten Bevölkerungsdichten in der Welt. Der führende Sektor des Wachstums wurde die Exportindustrie und das exportierende Dienstleistungsgewerbe, die durch relativ billige Lohnkosten und offenbar einer effizienten Organisation komparative Vorteile zu erzielen wußten. Damit finden wir hier so ziemlich alle Variablen vertreten, die von der modernen sozialwissenschaftlichen Entwicklungstheorie als Bedingungen und Ursachen für ,, Wachstum ohne Entwicklung“ bzw. ,Entwicklung der Unterentwicklung“ in den peripherkapitalistischen Staaten diagnostiziert worden sind. Haben wir es also auch hier wiederum nur mit einer derartigen Fehlentwicklung zu tun, die möglicherweise bald an ihre strukturellen Schranken treffen wird?

II.

Die Studie von Max Eli1 ist hier optimistisch. Für ihn beginnt Südkorea - nach einer überaus erfolgreichen Aufbauphase - nun in den Status eines NIC's (,,new industrialized country“) hineinzuwachsen. Allerdings kennt Eli kaum die Diskussion um den Unterschied zwischen Wachstum und Entwicklung. Ihm genügen die (konventionellen) Wachstumsindikatoren, die er detailliert nach Struktur und Wachstum des BSP, nach Entstehungsbereichen und nach den Verwendungsbereichen, untersucht. Als ehemaliger Wirtschaftsjournalist, der gegenwärtig in der Versicherungswirtschaft tätig ist, schrieb er dieses Buch allerdings nicht nur für den ,,akademisch Interessierten“, wie er im Vorwort sich ausdrückt, sondern auch für ,,den Praktiker aus Industrie und Außenhandel mit Investitionsabsichten in Korea." Insofern tut man Eli wohl Unrecht, wenn man seine Schrift auf die entwicklungstheoretisch relevanten Fragestellungen und Aussagen abklopft; sich zu diesen zu äußern, war primär offenbar gar nicht seine Absicht.

So reicht ihm z. B. eine halbe Seite (plus eine Tabelle) zur Darstellung der Verteilung des Volkseinkommens, wobei er im übrigen ,,auf die entsprechende Fachliteratur" verweist (sonst ist allerdings sein Materialreichtum gelegentlich etwas überladen und ermüdend) (S. 124). Fürdie Darstellung der Entwicklung der Monatsverdienste (offenbar der abhängig Beschäftigten) begnügt er sich auch nur mit den letzten Jahren (1974-77), gibt diese zudem nur in den nominalen, nicht realen (inflationsbereinigten) Werten an (sie scheinen dennoch für diesen Zeitraum erheblich zugenommen zu haben) (S. 154). Im allgemeinen bemüht er sich allerdings dankenswerterweise, die Wirtschaftsentwicklung in langen Zeithorizonten (und

1 Max Eli: Wirtschaftliche Entwicklungsperspektiven der Republik Korea. Hamburg: Mitteilungen des Instituts für Asienkunde Nr. 109, 1979, 180 S. DM 
immer auch mit den wirklich letzten Daten) zu erfassen. Für potentielle Investoren sind aber nun einmal nur die gegenwärtigen Löhne - und nicht die der Vergangenheit - interessant. Allerdings dürfte auch diese Klientel mit diesen Angaben sich nicht ganz erschöpfend bedient fühlen.

Die Löhne und Lohnkosten sind im asiatischen Kontext inzwischen schon relativ hoch (und werden nur noch von Japan übertroffen, vgl. Tab. S. 155). Der „Praktiker“ (d. h. Unternehmer) weiß aber, daß niedrige Löhne nicht unbedingt ,,billig“ sind, oder - marxistisch ausgedrückt - die Aneignung einer hohen Mehrwertrate erlauben. Diese hängt vielmehr auch von der Arbeitsproduktivität (aufgrund der Arbeitsintensität) ab. Relativ hohe Löhne können also durchaus noch vergleichsweise höhere Gewinne ermöglichen.

III.

Ähnlich euphorisch wie Eli sehen auch Wade/Kim² die südkoreanische Entwicklung. Sie bemühen sich allerdings um eine entwicklungstheoretische Diskussion. Ihre Daten - die sie häufig in statistischen Tabellen mit anderen Ländern vergleichen - reichen jedoch meist nur bis 1972/73. Für sie handelt es sich bei Südkorea um ,,eine politische Okonomie des Erfolges, eines das nicht unter der Peitsche des Totalitarismus oder vom Wunschdenken eskapistischer Utopisten abhängt.“ (S. VIII). Hier sei es gelungen, eine ,,industrialisierte und zunehmend reiche moderne Gesellschaft" aufzubauen (S. VI). Dieser Erfolg sei zu einem großen Teil ,,der Konsolidierung und Stabilisierung der politischen Macht und der Entschlossenheit mit welcher die Politik von der politischen Führung mit einer wachsenden administrativen Kapazität implementiert worden sei, zu verdanken“ (S. 240).

Nicht nur diese genannten Zitate lassen bei dem Leser gelegentlich den Verdacht aufkommen, daß es sich auch bei dem vorliegenden Werk um eine von der Regierung bezahlte Auftragsarbeit handelt, mit der diese sich verdeckt Zugang zum wissenschaftlichen Buchmarkt zu eröffnen versucht. Es handelt sich übrigens um eine Praxis, der sich autoritäre Regime in Entwicklungsländern nicht selten bedienen.

Wade/Kim behandeln die Entwicklung der Investitions- und Sparrate, die Investitionseffizenz (ICOR), die Steuermobilisierung, die Exportentwicklung, das Bildungswesen, das Bevölkerungswachstum und die Verteidigung - auf ökonomischer Ebene -, schließlich auch Probleme und Strategien der staatlichen Planung und administrativen Umsetzung sowie des politischen Willensbildungsprozesses wirtschaftspolitischer Entscheidungen. Wie schon bei Eli sind die Informationen über die staatlichen Lenkungsmaßnahmen allerdings nicht sehr konkret und damit auch nicht in ihrer Detaileffizenz abschätzbar. So hätte man auch gern Genaueres erfahren über das Verhältnis der internen zu den Exportpreisen (Dumping) und natürlich den Austauschbeziehungen zwischen dem Agrar- und dem urbanen Sektor: Auch wird kaum diskutiert, wer (unternehmerischer) Träger dieses Wachstumsmodell ist: der Staat, die MNK oder die nationalen Bourgeoisie. Die letztere wird hierbei sicherlich zunächst die schwächste Rolle spielen. Von zentraler entwicklungstheoretischer Bedeutung wäre aber zu wissen, wie ihre Entwicklungsperspektiven sind bzw. vom Staat gestaltet werden: Wird sie noch weiter verdrängt, kann sie in Gestalt ausgewählter Individuen durch besondere Beziehungen zum staatlichen Lenkungs-, Subventions- und Distributionsapparat

2 Wade, L. L./Kim, B. S.: Economic development of South Korea. The political economy of success. New York: Praeger 1978,270 p. $14,25 £$. 
eine parasitäre Existenz führen oder ist der Staatsapparat tatsächlich in der Lage, Geburtshelfer einer sich entwickelnden genuinen nationalen Bourgeoisie zu sein?

Auch sollte man die Frage der Technologie und die Entwicklung der eigenen innovativen Fähigkeiten einer Gesellschaft nicht aussparen, wenn man deren Eintritt in den Status eines reichen industrialisierten Landes feiert, das - nun allerdings noch einmal in der Formulierung von Eli (S. 157) - ,, in die Verantwortung für die Stabilität der Weltwirtschaftsbeziehungen einbezogen wird“.

Der Rezensent ist hier nicht der Meinung, daß man schon in einem frühen Entwicklungsstadium nach eigenständigen Technologien und nur örtlich entwickelten Neuerungen als Meßlatte zur Beurteilung einer Entwicklungsstrategie schauen soll. Diesbezügliche Versuche (etwa in China) werden häufig (von Dependenztheoretikern) überschätzt bzw. überbewertet. Gerade in einem frühen Entwicklungsstadium kann es sehr sinnvoll sein, Technologien nicht selbst zu entwickeln, sondern zu importieren. Bedenklich ist allenfalls, wenn autochton vorhandene Kenntnisse und Fähigkeiten verschwinden oder verdrängt werden, obwohl sie eigentlich ihre Funktion noch recht gut hätten erfüllen können. Aber auch die Handhabung der importierten Technologie bereitet bekanntlich in nicht wenigen Entwicklungsländern Schwierigkeiten, die ihre Effizienz und entwicklungspolitische Wirksamkeit oft einschränkt und ihre volks- und betriebswirtschaftlichen Kosten nach oben treibt. Es wäre hier sinnvoller zu fragen, in welcher Weise eine Entwicklungsgesellschaft in der Lage ist, aus der vielfältigen Palette international verfügbarer Technologie diejenigen Verfahren und Patente auszuwählen und zu nutzen, die ihren eigenen Ressourcen, Bedürfnissen und Zielen am angemessensten sind. Weiter wäre zu prüfen, in welcher Weise eine Gesellschaft fähig ist, diese importierte Technologie in eigenen Produktionsverfahren in national kontrollierten und geführten Betrieben, in welchem Umfang mit nationalen technischen und administrativen Kadern zu nutzen. Erst wenn dies voll gelingt (etwa auch in dem Sinne, daß Patente gestohlen und nachgebaut bzw. freiwerdende Patente umgehend genutzt werden), sollte man erwarten, daß auch langfristig konkurrenzfähige internationale Technologie selbst entwickelt wird.

In keiner Studie über den Entwicklungsweg eines Landes sollte die kritische Frage versäumt werden: Wer partizipiert, in welchem Umfang, mit welchem (ökonomischen) Nutzen? Das heißt, es ist dabei zu fragen, in welcher Weise bisherige Produktionsweisen bzw. Branchen verdrängt, in marginale Positionen abgedrängt oder auch stimuliert, am Wachstumsprozeß beteiligt oder - wenn sie als Produktionsform aufgehoben werden (etwa der subsistent wirtschaftende Familienhaushalt) - ob seine Mitglieder in andere Form in den dynamischen ökonomischen Prozeß integriert werden und in der einen oder anderen Weise an ihm (auch für sie positiv) partizipieren. Über diese strukturellen Veränderungen und ihre Konsequenzen z. B. Verhältnis der (modernen) großen zu den (,,traditionalen“) kleinen Betrieben, der Subsistenz- zur Marktökonomie, der Agrar- zu den urbanen Produzenten, der kleinen Warenproduzenten zu den Lohnarbeitern - geben Wade/Kim, wie auch schon Eli - kaum Auskunft.

Immerhin lassen sie die Einkommensebene nicht ganz außer acht. Dabei bejahen sie die definitorische Notwendigkeit, Wachstum und Entwicklung auch durch den Anstieg der Masseneinkommen zu messen (S. 38). Die Veränderung und Entwicklung der privaten Einkommen kann mindestens durch drei Komponenten erfolgen: durch den Anstieg der Realeinkommen der Beschäftigten, durch die Zunahme der produktiven Beschäftigung ganz allgemein und schließlich durch die größere und bessere Nutzung kollektiver Güter.

National aggregierte Daten - Nationalprodukt p.c., Ärzte usw. p.c., Anzahl der Kraftfahrzeuge, Telefone, Radios, TVs, usw. (bei Wade/Kim, S. 28, 43 ff.) - sind in sozial stratefi- 
zierten Gesellschaften nur beschränkt aussagefähig. Sie belegen allenfalls, daß in einer Gesellschaft Wachstum stattgefunden hat, nicht jedoch, wer, in welchem Umfang an ihm partizipiert hat; also z. B. ob nur eine schmale Bourgeoisie, eine kleine und vielleicht schnell wachsende Mittelklasse oder auch die abhängig beschäftigten Lohnarbeiter sowie die kleinen Warenproduzenten usw.

Um dieses Problem in den Griff zu bekommen, ist die Untersuchung der Einkommensverteilung sehr beliebt. Auch Wade/Kim tun dies (S. 77 ff.). Obwohl sie ihre Skepsis gegenüber diesen Daten nicht verhehlen, fühlen sie ihre Argumentation durch diese bestätigt und argumentieren daher mit ihnen: Südkorea würde demnach mit zu den Ländern gehören, in denen die Ungleichheit der Einkommen noch mit am relativ geringsten ausgeprägt ist, obwohl dies ein ,,potentielles Problem“ "bleibe (S. 81). Der Rezensent hält die Beobachtung der Einkommensunterschiede für sinnvoll, aber auch in der Bedeutung überschätzt. Einmal handelt es sich hierbei um ein Gebiet, auf dem es wohl am schwierigsten ist, einigermaßen verläßliche Daten zu erhalten. Auch ist das Postulat der Einkommensgleichheit und die kapitalistische Organisation einer Gesellschaft ein Widerspruch. Am ersteren kann allenfalls statistisch herummanipuliert und können besonders krasse Spitzen egalisiert werden (wie es der Staat im hochentwickelten Kapitalismus durch seine Steuer- und Distributionspolitik z. T. versucht). Eine volle Egalisierng impliziert aber letztlich eine Aufhebung dieses Systems (woran Nicht-Marxisten in der Regel wohl nicht gedacht haben werden). Dies bedeutet nicht, daß die Lohnempfänger in der politischen Praxis ihre Lohnforderungen etwa nicht an die Profitentwicklung orientieren sollten. Diese Argumentation bedeutet gleichfalls nicht, daß man über öffentliche internationale Entwicklungshilfe Tendenzen zur Einkommensegalisierung durch gezielte Unterstützung von Armutsgruppen nicht zu fördern versuchen sollte, was theoretisch auch immer wieder beschworen, in der Praxis allerdings so selten erreicht wird. Man sollte nicht in erster Linie die relative Gleichheit oder Ungleichheit der Einkommen für Erfolg oder Mißerfolg eines Systems und Entwicklungsweges beobachten, sondern die Entwicklung der absoluten Höhe der Masseneinkommen. Wenn auch die ärmeren Schichten zur Befriedigung ihre Grundbedürfnisse (zunehmend besser) in der Lage sind und darüber hinaus ein reales Wachstum ihrer Einkommen zu verzeichnen ist, so ist dies wichtiger als die Feststellung, daß der Abstand zu den Oberschichten dennoch noch größer geworden ist. Dies trifft um so mehr zu, wenn die These über die Inkompatibilität zwischen Einkommensgleichheit und Wachstum zuträfe: Nämlich wenn die Egalisierung der Einkommen zu einem allgemein niedrigen (gleichmäßig zu verteilenden) Nationalprodukt führt. Vorzuziehen wäre dann doch wohl ein System, das die Produktivkräfte ,,entfesselt" und letztlich allen, wenn auch ungleich, zugute kommt. Nun ist diese Situation in den peripher-kapitalistischen Entwicklungsländern heute oft nicht gegeben: Wirtschaftswachstum ist hier oft mit einer krassen Einkommensungleichheit und nicht nur relativ, sondern auch absolut zunehmender Massenarmut verbunden. Ob es sich im konkreten Einzelfall (etwa in Südkorea) auch um dieses Modell handelt, wäre natürlich im einzelnen zu untersuchen. Die hier angezeigten Bücher leisten dazu allerdings keinen befriedigenden Beitrag.

Sinnvoll wäre es, beispielsweise, die Zunahme der nicht-agrarischen Lohnarbeitsverhältnisse zu registrieren. Sie gibt Aufschluß darüber, ob die kapitalistische Produktionsweise sich tendenziell verallgemeinert oder eben sich selbst blockiert; ob der urbane (industrielle) Bereich in der Lage ist, die agrarische Surplus-Bevölkerung sowie den laufenden Bevölkerungszuwachs abzuziehen und produktiv einzusetzen und damit potentiell eine höhere Pro-Kopf-Produktivität auch in der Landwirtschaft ermöglicht. Gelegentlich werden die Daten es erlauben, die Entwicklung der Lohnarbeiterschaft nicht nur als Globalgröße zu verfolgen, sondern auch in ihrer Zusammensetzung zu analysieren: Nimmt sie nur in der 
Groß- oder auch in den Handwerksbetrieben zu, stagniert sie etwa in den letzteren aufgrund der Expansion der ersteren; findet sie im privaten oder auch öffentlichen Sektor statt, vielleicht im letzteren bei sinkender Produktivität pro Kopf ${ }^{3}$. Schließlich wären genauere Informationen über die Beschäftigung selbst wichtig: Handelt es sich um permanente Arbeiter (oder sind sie nur saisonal bzw. sporadisch beschäftigt), ist die Arbeiterschaft geschlechtsund altersspezifisch relativ ausgewogen (oder wird z. B. nur Arbeit für junge Frauen angeboten); werden von den Arbeitern schon besondere Qualifikationen und Fertigkeiten mitgebracht bzw. werden diese verlangt oder handelt es sich überwiegend um eine Anstellung von ungelernten bzw. angelernten Arbeitern?

Will man Aussagen über die Entwicklung der Masseneinkommen machen, ist es sinnvoll, einzelne - relativ homogene - gesellschaftliche Gruppen zu betrachten. Am einfachsten werden immer Daten über die Lohneinkommen verfügbar sein (sowohl über die Nominalwie auch die [inflationsbereinigten] Realeinkommen). Hier ist es interessant, die Lohnentwicklung für akademisch und technisch gebildete Angestellte und ungelernte Arbeiter zu vergleichen. In typischen Entwicklungsländern ,,mit unbegrenztem Angebot an unqualifizierter Arbeit" finden wir hier extreme Unterschiede.

Schwerer sind einigermaßen verläßliche Daten (auch über längere vergleichbare Perioden) für die kleinen Warenproduzenten, also Handwerker, Händler usw. in der Stadt, Klein- und Marginalbauern auf dem Lande, zu erhalten. Bei einer relativ homogenen Agrarstruktur, wie wir sie in Südkorea antreffen, wo der weitaus größte Teil der Anbaufläche von Betrieben unter 3 ha kontrolliert wird, kann eine Beobachtung der (meist verfügbaren) Makroindices schon einigen Aufschluß geben (Agrarbevölkerung, Agrarproduktion, Input- und Outputpreise, Verfügbarkeit institutionellen Kredits). Für den informellen Sektor der Städte ist dies weit schwieriger: Hier wird man an detaillierten empirischen Sample-Untersuchungen nicht vorbeikommen, wenn man irgendwie abgesicherte Aussagen machen und nicht allein nur mit den Residualgrößen von Makroindices arbeiten will.

IV.

Anspruchsvoller und abgewogener als die beiden bisher vorgestellten Arbeiten ist die Monographie von Paul W. Kuznets ${ }^{4}$, wenn auch diese längst nicht voll befriedigen kann.

Nach einem etwas längeren historischen Rückblick behandelt er die Gründe und Konsquenzen des beschleunigten Wachstums, untersucht das Problem der Arbeitsbeschaffung, die Funktion und Entwicklung des Agrarsektors, des Industriesektors, schließlich die Preisentwicklung und Geldpolitik sowie das Zustandekommen und die Ziele der drei ersten Fünfjahrespläne. Obwohl er einleitend die Zielsetzung seiner Arbeit dahingehend einschränkt, daß er hauptsächlich die ,,ökonomischen Beziehungen und weniger die politischen Fragen . . . primär auf quantitative Ebene" bringen will (S. XII), wird aus seiner Studie der $\mathrm{Zu}$ sammenhang von Politik und Okonomie viel deutlicher als bei den Arbeiten von Eli und Wade/Kim. Er zeigt so z. B., daß die prioritäre Förderung der Industrialisierung durch den Staat durch die (politische) relative Verbilligung der Kapitalkosten erfolgte, die insbesondere die großbetrieblichen und kapitalintensiven Unternehmen förderte. Man bediente sich dabei der Zoll-, Währungs- und Importlizenzpolitik, stellte relativ billigen Kredit zur Verfügung

3 In diesem Fall würde es sich also nur um ein politisches Arbeitsbeschaffungsprogramm handeln, dem kein natürliches Wachstum der Prokuktivkräfte entspricht und das von der Allgemeinheit getragen werden muß.

4 Kuznets, Paul, W.: Economic groth and structure in the Republic of Korea. New Haven, London: Yale University Press, 1977, 238 p., $11,90 £$. 
und kontrollierte die Lohnkosten. Letzteres wurde - nach Kuznets (und hierbei ist er nicht erschöpfend) - erreicht durch billige Nahrungsmittelimporte, durch Agrarförderungsprogramme (durch Bereitstellung billigen Düngers usw.) in Verbindung mit der Kontrolle der Nahrungsmitteleinzelhandelspreise und schließlich durch die Einschränkung der Bewegungsfreiheit von Gewerkschaften und von legalen Lohnkampfmaßnahmen (S. 121). "Because this regime has been authoritarian and has no economic interest base, it could hold down wages and consumption, largely ignore rural interests, and concentrate on rapid development, in turn fostered sectoral and geographic imbalances and possibly growing income inequality.” (S. 85). Dennoch meint auch Kuznets, daß die ,Durchschnittsperson“ in Südkorea am Ende der zweiten Dekade, ,besser ernährt und besser gekleidet ist sowie besser wohnt" als zu Beginn der ersten (S. 92). Allerdings glaubt er nicht an eine wachsende Gleichheit der Einkommen, sondern nimmt eine wachsende Ungleichheit an. Die das Gegenteil belegenden Studien unterzieht er dabei einer sachlichen Kritik (S. 96 ff.).

Ausführlich beschäftigt er sich auch mit der Frage, in welchem Umfang Arbeitsplätze geschaffen wurden (S. 111 ff.). Für die Dekade 1963-72 errechnete er eine Zunahme der wirtschaftlich aktiven Bevölkerung um $33 \%$, wenn man die Zunahme der Intensität der Arbeit miteinbezieht, sogar um $48 \%$ - bei einem Bevölkerungswachstum von nur ca. $20 \%$ (S. 116). Dies wäre durchaus kein schlechtes Ergebnis. Allerdings macht Kuznets die z. Z. noch sehr hohe saisonale Abhängigkeit der Beschäftigung deutlich (S. 113). Auch schätzt er, daß 1972 noch ca. 1/4 des verfügbaren Arbeitskräftepotentials nicht genutzt wurde, d.h. arbeitslos und unterbeschäftigt war (S. 127). Obwohl auch die Arbeit von Kuznets in vielen Fragen durchaus noch detaillierter und umfangreicher empirisch belegt sein könnte, sie auch nicht frei von Fehlern ist, bietet sie jedoch eine relativ brauchbare Einführung zur ökonomischen Entwicklung des modernen Koreas. Allerdings hat die Arbeit die Entwicklung nur bis etwa 1972 berücksichtigt. Inzwischen ist gerade auch in Korea sehr viel geschehen.

V.

Der von Sung-Jo Park, Taiwhan Shin und KiZumZo5 herausgegebene Sammelband enthält 23 Beiträge über so nahezu alle Aspekte der ökonomischen und gesellschaftlichen Entwicklung des Landes. Die Artikel werden in sechs Kapitel untergliedert: Characteristics and determinants of economic growth, sectoral developments and structural barriers; manpower, employment, and labor market; foreign trade, imvestment, and regional cooperation; social aspects of economic development; social groups and their dynamics. Bis auf einen der Herausgeber, Sung-Jo Park, der Profesor an der FU Berlin ist, sind alle anderen Auroren Hochschullehrer in Südkorea. Entsprechend angepaßt ist - durchgängig - ihre Argumentation. Regierung und Politik des Parkregimes, das zum Zeitpunkt der Abfassung der Manuskripte sich noch an der Macht befand (die Daten reichen übrigens meist nur bis 1975/76), wird kaum kritisch durchleuchtet oder gar kritisiert. Hauptquelle sind amtliche Statistiken, die aber unkritisch und ohne Diskussion kommentiert werden. Viele bekannte Fakten werden einfach unterschlagen. Regierungsprogramme - auf die das Regime selbst besonderen propagandistischen Wert legte - werden als besonders erfolgreich hochgejubelt, ohne daß nur der Versuch unternommen würde, die Kriterien der Bewertung und die Methode der Mes-

5 Sung-Jo Park, Taiwhan Shin, Ki Zun Zo (eds.): Economic development and social change in Korea. Frankfurt, New York: Campus Verlag 1980, 400 p., DM 48,- 
sung darzulegen. Dazu einige Beispiele: Die Politik der Regierung, die Produzenten und damit auch die Verbraucherpreise für Grundnahrungsmittel niedrigzuhalten, wird in einem Beitrag nur kurz erwähnt (Soon Cho, p. 40: "Policy of maintaining low agricultural prices . . . to prevent inflation and to subsidize the urban population"). Zwei andere Autoren berichten darüber nur als diese Politik vorübergehend abgeschwächt wurde. Bei Ki Hyuk Pak.(S. 58), heißt es so nur ,,traditional low farm prices were phased out during the 1970's“. Eine Tabelle, die nur die nominalen (nicht inflationsbereinigten) Steigerungen zeigt, läßt diese tatsächlich gewaltig erscheinen (S. 60). Ein anderer Autor (Sang Chul Sul, p. 122) spricht ähnlich wiederum nur davon ,,The government's price policy for farm products has recently been an important instrument for uplifting rural income level." $\mathrm{Da}$ die staatlichen Aufkaufpreise für einen Teil des Getreides schon einmal mehr als die Hälfte unter den Produktionskosten lagen, wird nicht erwähnt. Auch die Saemaul-Undong-Bewegung findet allenfalls lobende Erwähnung und euphorische Würdigung (S. 10, 51, 70/72, 122), als ob es da nun gar keine kritischen Aspekte zu diskutieren gäbe. Die Notwendigkeit von Mindestlohnerhöhungen wird bestritten und die Knebelung und Manipulation der Arbeitsvertretungen in folgenden Worten umschrieben: "But, because labor union activity is not yet satisfactory, it is difficult to expect successful income redistribution through labor unions and thus it seems inevitable to coordinate wages through governmental policy or by spontaneous increase in wages by enterprises, even though such measures are not considered most effective" (Taiwhan Shin, p. 12). Hier werden also Ursachen und Wirkung munter miteinander vermischt und die Absichten der Beteiligten vernebelt ${ }^{\mathbf{6}}$.

Natürlich sind auch alle Autoren optimistisch über die weiteren Wachstums- und Entwicklungsaussichten ihres Landes (u. a.: p. 14, 53, 228). Diese Liste mit Mängeln, Fragwürdigkeiten und Auslassungen ließe sich noch erheblich verlängern, darauf soll aber verzichtet werden. Es bleibt nur, die koreanischen Kollegen zu bedauern, die offenbar unter derartig repressiven Bedingungen Sozialwissenschaft betreiben müssen. Mag dieses Buch auch nicht ganz wertlos sein und die eine oder andere ,,offiziöse” Information enthalten, so bleibt doch die Frage im Raum, warum ausgerechnet der sonst gesellschaftskritische Literatur führende Campus-Verlag dieses Erzeugnis veröffentlicht.

VI.

Die Studie von Suck-Kyo $\mathrm{Ahn}^{7}$ behandelt nur einen - wenn auch gerade für dieses Land wichtigen - Teilbereich der hier erörterten Thematik: die Entwicklungsfunktion ausländischer Direktinvestitionen in Korea. Sie versteht sich dabei z.T. auch als eine empirische Teilantwort auf die weithin einflußreiche Arbeit von Fröbel/Heinrichs/Kreye über die „,Neue internationaleArbeitsteilung" als Konsequenz der Auslagerungsindustrien der MNK in Exportzonen der Entwicklungsländer. Theodor Dams schreibt in seiner lesenswerten Einleitung (die Auskunft über den Diskussionsstand der Kirchen und christlicher Kreise in dieser Frage gibt), daß wenn die Ergebnisse und Schlußfolgerungen dieser besagten Studie zuträfen, ,,Auseinandersetzungen über die marktwirtschaftliche Ordnung in den internationalen Wirtschaftsbeziehungen" unausweichlich würden (S. VIII). Die Studie von Fröbel et. alii.

\footnotetext{
6 Immerhin wird im Beitrag über die Gewerkschaften erwähnt, daß Polizei und Geheimdienst (KCIA) Arbeitskonflikte ,,am effektivsten" schlichten und daß seit 1972 die einzig legitime (gemeint ist legale) Rolle der Gewerkschaften darin besteht, Regierungsprogramme zu unterstützen und Petitionen einzureichen (Young Ki Park, p. 394, 396).

7 Suck-Kyo Ahn: Ausländische Privatinvestitionen in Südkorea. Vorauss etzungen und Auswirkungen auf die sozialökonomische Entwicklung - Eine Fallstudie. Mainz, München: Kaiser, Grünewald 1980, 154 S., DM 19,50.
} 
sei hier allerdings noch nicht schlüssig, da ,,normativ nur ein bestimmtes Material zum Nachweis herangezogen" worden wäre (eine Auffassung, die auch der Rezensent teilt). Der Brückenschlag zwischen ,,,großer Theorie“ und , grober Empirie'” müsse also in vielen Einzelstudien noch geleistet werden. Die Arbeit von Suck-Kyo Ahn stellt hierfür in der Tat einen wichtigen Beitrag dar. Er bemüht sich mit Erfolg, die pros und cons der ausländischen Direktinvestitionen in Südkorea zusammenzutragen. Er verzichtet dabei auf jeglichen rhetorischen Ballast und ideologische Aggressivität, mit der andere Autoren immer wieder ihre dünne Decke gesicherter empirischer Fakten zu umnebeln versuchen. Gelegentlich wirkt sein Stil dabei allerdings doch zu unterkühlt. Schon aus didaktischen Gründen hätte man sich vielleicht gelegentlich eine pointierterte Hervorhebung und Diskussion der Einzelergebnisse gewünscht. Die Studie besteht aus zwei Teilen. Zunächst wird die Entwicklungssituation als „,sozio-ökonomische Vorraussetzungen der ausländischen Direktinvestitionen in Korea” beschrieben. In weniger als 30 Seiten werden hier die wesentlichen Punkte kompetent aufbereitet und angesprochen: die staatlichen Entwicklungsstrategien, die relevanten wirtschaftlichen Indikatoren (sektorale Struktur, Exportleistung, Verschuldungssituation, Beschäftigung, Produktivität, Löhne), die Bildungssituation und die Einkommensverteilung. Wer sich in Kürze zu diesen Fragen informieren will, der sollte schon zu der vorliegenden Schrift greifen. Den größeren Raum der Arbeit nimmt die Darstellung der staatlichen Politik und Förderungsmaßnahmen gegenüber den ausländischen Direktinvestitionen, deren Struktur sowie deren sozio-ökonomischen Konsequenzen ein (ca. 80 S.). Für diesen Teil hat der Autor in über 34 ausländischen Unternehmen eigene empirische Daten erhoben, auf die er sich im folgenden im wesentlichen stützt. Er kommt dabei zu dem Schluß, daß der Beschäftigungseffekt der ausländischen Investitionen beachtlich ist, dabei ist er noch relativ größer in den Exportindustrien als in den für den Binnenmarkt produzierenden Unternehmen (S. $71 \mathrm{ff}$.). Allerdings ist der Anteil der (schlecht bezahlten) weiblichen Arbeitskräfte überproportional hoch. Die Sicherheit der Arbeitsplätze in den weltmarktabhängigen Exportindustrien (anders als in den binnenmarktorientierten Industrien) ist sehr fragil, was u.a. auch nach der ersten Energiekrise (1973/74) zu vielen Entlassungen, dann aber zum eingefrorenen Abbau der Arbeitsplätze (durch Schicht- und Úberstundenarbeit usw.) führte (S. 78/79). Die Lohnstruktur ist sehr differenziert. Im Durchschnitt liegen die Löhne in den ausländischen Unternehmen zwar über denen in den koreanischen Betrieben; sie liegen hier aber in den exportorientierten Unternehmen (in den Freihandelszonen) erheblich unter denen der binnenmarktorientierten Unternehmen (in Seoul) und wohl z.T. auch unter denen der einheimischen Unternehmen. Erhebliche Unterschiede gibt es zudem es in der Entlohnung der männlichen und weiblichen Arbeitskräfte sowie zwischen den Hochschul- und den Mittelschulabsolventen (S. 79 ff.). Die Löhne erlaubten zwar kräftige Steigerungsraten (der Autor bezieht sich allerdings nur auf die Jahre 1973-76), die jedoch durch eine fast ebenso hohe Preisentwicklung nahezu neutralisiert wurden, so daß die Reallohnsteigerungen relativ bescheiden und erheblich unter der durchschnittlichen Steigerungsrate der Produktivität blieben.

Allerdings sind die Löhne im Agrarbereich - im Zuge einer zunehmenden Verknappung der Arbeitskräfte auf dem Lande - erheblich gestiegen und haben sich (1976) in etwa denen in den Freihandelszonen angeglichen (S. 92). Die Landflucht erklärt der Autor daher eher sozial als ökonomisch motiviert (S. 93) Die Ausbildungs- und Anlernaufwendungen der meisten untersuchten Unternehmen sind sehr gering. Bei den ungelernten Arbeitern erfolgen sie zudem weitgehend auf deren Kosten. Für Fachkräfte wurden sie in vielen Unternehmen inzwischen erheblich eingeschränkt, da diese (aufgrund der niedrigen Löhne und wegen der Unsicherheit der Arbeitsplätze) meist allzu rasch in koreanische Betriebe abwanderten. 
Auch über den angestrebten und erhofften Technologietransfer läßt sich kaum Positives sagen: Forschung findet in den ausländischen Unternehmen selbst kaum statt. Auch die Vermarktung erfolgt überwiegend durch die japanischen und amerikanischen Mutterhäuser. Diese behalten damit auch die Marktübersicht und das Monopol der Vermarktungstechnologie - ein Punkt, der für den dauernden Nutzen und die dauernde Existenz dieser Betriebe in Korea von ausschlaggebender Bedeutung ist, wenn die ausländischen Unternehmen einmal abziehen bzw. herausgedrängt werden sollten.

Die Auswirkungen auf die Zahlungsbilanz sind zwar positiv, die Absatzstruktur wurde auch erheblich diversifiert, jedoch werden überdurchschnittliche Gewinne gemacht, die die Investitionen schon in wenigen Jahren amortisieren und überwiegend retransferiert (und nicht re-investiert) werden (S. 113 ff.). Als Negativposten wird noch die Umweltverschmutzung, die praktisch unkontrolliert und ohne nennenswerte Sicherheits- und Schutzauf wendungen erfolgt, genannt (S. 128 ff.) und die Problematik der für den Binnenmarkt produzierenden Düngemittelfabriken unter multinationaler Kontrolle in ihren wesentlichen Einzelheiten erörtert (S. 123 ff.).

Dies ist sicherlich ein ernüchternder Befund, auch wenn methodisch gelegentlich noch einige Fragezeichen gesetzt werden müssen bzw. relevante Daten noch nicht verfügbar waren ${ }^{8}$. Suck-Kyo Ahns Schlußfolgerungen und Handlungsanweisungen (für den koreanischen Staat) bleiben systemimmanent. Er befürwortet nicht eine radikale Kehrtwendung in der Politik gegenüber den MNK, sondern deren gezieltere Förderung, eine bewußtere Politik gegenüber der Desinvestitionspolitik der ausländischen Unternehmen, ihre partielle Ablösung durch andere Modelle der Zusammenarbeit (Lizenzverträge), die Ausbildung von qualifizierten Experten um die Effizienz des staatlichen Lenkungsapparates zu verbessern (S. 133 ff.).

Seine eher moderaten Vorstellungen - die man sich allerdings durchaus noch erweitert vorstellen könnte - sollten aber nicht durch den Umstand erklärt und damit abqualifiziert werden, daß Suck-Kyo Ahn sich schließlich nicht seine Karriere in seinem Heimatland verbauen wollte (er ist inzwischen Professor an der Han-Yan-Universität Seoul), sondern sollten als der durchaus sinnvolle Versuch verstanden werden, durch einigermaßen praktikable und akzeptable Vorschläge, sich die Chance einer politischen Einflußnahme offenzuhalten (um eben nicht nur für die ,, akademische Community” im Elfenbeinturm zu produzieren). Andererseits kann auch nicht übersehen werden, daß dem sozialpolitisch und nationalökonomisch orientierten Lenkungspotential der Staaten in der Dritten Welt gegenüber MNK (im Industriesektor) solange relativ enge Grenzen gesetzt sind, wie es nicht gelingt, im regionalen und überregionalen Rahmen zu einer konzertierten Politik der Anlageländer zu kommen (etwa für Ost- und Südostasien im Rahmen der ECAFE; in der UN: ,,Code of Conduct”). Davon sind wir allerdings, trotz einiger unverbindlicher Ansätze, noch weit entfernt. Die Konkurrenz zwischen den einzelnen Anlageländern - zu denen sich nun auch noch die VR China hinzuzugesellen versucht - ist heute noch das bestimmende Element.

\footnotetext{
8 So reicht der Nachweis einer bestimmten Beschäftigtenzahl in ausländischen Unternehmen zur Quantifizierung ihres Beschäftigungsbeitrages natürlich nicht aus, die alternative Verwendung der öffentlichen Vorleistungen (etwa in den Industriezonen) müßte hier auch berücksichtigt werden usw.
} 
VII.

Auch den Autoren des von Du Yul Song ${ }^{9}$ herausgegebenen Sammelbandes kann man gewiß nicht nachsagen, daß sie den Unterschied zwischen Wachstum und Entwicklung nicht kennen würden. Bei ihnen handelt es sich überwiegend um linke Autoren, die den Charakter und die Konsequenzen der kapitalistischen Weltarbeitsteilung im allgemeinen und der gegenwärtigen Rolle Südkoreas in ihr (für die dort arbeitenden Menschen) kritisch gegenüberstehen.

Von den acht Beiträgen behandeln zwei (von M. Y. Cho und Bruce Cummings) außenpolitische Probleme sowie ein weiterer die Rollen der Mun-Sekte (von Sek-Keun O/Du-Jul Song). Sie sind für den Zusammenhang unserer hier geführten Diskussionen nicht so ergiebig und sollen daher nicht weiter gewürdigt werden. Zwei Beiträge versuchen einen Vergleich zwischen Nord- und Südkorea (zur Entwicklungsstrategie: Du Yul Song; zur Rolle der Ideologie und von Massenkampagnen: Albrecht Lein); zwei weitere beschränken sich auf Einzelaspekte in Südkorea (zur Rolle der Regierungskampagnen: Hans U. Luther; der Arbeiterinnenbewegung: Ilse Lenz). Dieter Senghaas steuert eine entwicklungstheoretische Einleitung bei.

Die Autoren zeigen die andere Seite des (für sie sog.) südkoreanischen Wirtschaftswunders (das sie damit in Frage stellen): die Ausbeutung der Arbeiter(innen) und Bauern, die Technik ihrer Unterdrückung. Insbesondere der Beitrag von' Ilse Lenz über die Arbeitsbedingungen der Frauen, den Methoden ihrer Repression, die Versuche ihrer Selbstbefreiung, sei all denen zur Lektüre empfohlen (wie z. B. auch den hier eingangs vorgestellten Autoren), die allzu einseitig auf die markroökonomischen Indikatoren schauen und allein daraus ihre Schlüsse ziehen (daß eben Südkorea schon ein weit entwickeltes ,Schwellenland“ sei). Wie immer man den bisherigen Entwicklungsweg und die Entwicklungsperspektiven einschätzen mag, die Arbeitsverhältnisse und Arbeitsbeziehungen sind in vielfacher Hinsicht denen des Frühkapitalismus in Europa (des 19. Jahrhunderts) vergleichbar. Diese sind, gemessen an unserem Standard heute, unerträglich und waren es auch aus der Sicht der Betroffenen im Europa des 19. Jahrhunderts und der Arbeiter heute in Korea. Dieser Umstand war aber ein Grund, warum sich in Europa eine Arbeiterbewegung formierte und eine Verbesserung der Arbeitsverhältnisse und Realeinkommen der Lohnabhängigen mit erzwingen konnte. In welcher Weise dies z. B. auch in Südkorea (langfristig) möglich ist, bleibt abzuwarten bzw. wäre zu diskutieren. Unsere Sympathie sollte sicherlich bei den arbeitenden Menschen liegen, die, wo immer möglich, auch unsere Unterstützung erhalten sollten. Es muß aber sehr bezweifelt werden, ob wir ihnen durch die Produktion wissenschaftlich aufgemachter, politischer Pamphlete dabei wirklich helfen können, ob wir nicht als Wissenschaftler versuchen sollten, die Realität so exakt wie möglich als Grundlage einer prognostischen Analyse und politischen Handlungsleitung recherchieren sollten. Dies ist offenbar von einigen Autoren des vorliegenden Sammelbandes nicht beabsichtigt. Für sie liegt wohl das Ergebnis von Anfang an fest. Andere ernst zu nehmende Autoren bzw. anderweitig aufbereitete Tatsachen brauchten so von ihnen nicht berücksichtigt zu werden. Informationsdefizite werden einfach mit kühnen Spekulationen (die die eigene These ,,belegen“) überbrückt. Ursprungshinweise für mitgeteilte (oft fragwürdige) Fakten werden nicht selten ver-gessen. Beliebt ist auch die zeitliche Selektion von Zahlen-Daten, d. h. als Beleg einer These (für die Ursache einer Entwicklung) werden (u. U. durchaus zutreffende) Zahlen für ein,

9 Du-Yul Song (Hrsg.): Wachstum, Diktatur und Ideologie in Korea. Bochum: Studienverlag Dr. N. Brockmeyer 1980, 233 S., DM 24,80 . 
zwei Jahre angeführt, die eigentlich notwendigen Zahlenreihen für ein, zwei Jahrzehnte jedoch unterschlagen (oder war man einfach nur zu faul, sich um diese zu bemühen?).

Obwohl die hier versammelten Autoren z. T. in Details voneinander abweichen, z. B. Einschätzung der Seamaul-Bewegung (eher positiv systemimmanent: Lein, S. 65, völlig ablehnend: Luther), Entwicklung der Reallöhne (. . verschlechtern sich ständig: Luther, S. 103, . . sind leicht angestiegen: Lenz, S. 131), sind sie sich doch über die Fragwürdigkeit des südkoreanischen Modells und dessen Fehlentwicklung sowie explizit oder implizit über den Erfolg des nordkoreanischen Entwicklungsweges einig ${ }^{10}$.

Es ist im Rahmen dieser Rezension nicht möglich, auf alle relevanten und kontroversen Fragen einzugehen, die dieser Sammelband aufwirft. Dies würde auch etwas seine Bedeutung übertreiben. Daher sei die Kritik nur an einigen Beispielen erläutert.

So sind sich Du-Yul Song, Albrecht Lein und Hans Luther darüber einig, daß ,,noch nie in der koreanischen Geschichte“ die Bauernschaft so rasch ,,verelendete " wie in dieser Periode (so Lein, S. 64). Für diese These wird man in der gesamten (wegen mirauch ,,bürgerlichen“) Korea-Literatur kaum noch Anhänger finden. Es ist daher nicht unberechtigt zu erwarten, diese These empirisch substantiiert zu sehen.

Als Argument wird hier im wesentlichen die Landflucht angeführt (u. a. S. 26, 64, 89) und die Zunahme der Nahrungsmittelimporte (S. 27), beides als Ausdruck des weitgehend extern verursachten Realeinkommensverfalls und Motivationsverlustes der südkoreanischen Bauern.

In der Tat sprengte die Land-Stadt-Wanderung (vornehmlich nach Seoul) alle Maßstäbe. Allerdings wäre zunächst der Umstand, daß die koreanische Landwirtschaft die höchste Flächenproduktivität in Asien (nächst Japan) in den letzten Jahren erzielen konnte, nicht unbedingt ein Beleg der oben genannten These. Desgleichen der Tatbestand, daß wir es in Korea mit einer der höchsten Arbeitskräftedichten je Hektar in der Welt und daher auch mit den kleinsten Betriebseinheiten zu tun haben (alles Fakten, die im vorliegenden Sammelband nicht genannt und diskutiert werden $)^{\mathbf{1 1}}$. Der Subsistenzkonsum muß also schon hoch sein. Das Angebot von Beschäftigungsmöglichkeiten in der Stadt kann daher als ein erheblicher ,,pull'-Faktor für die chronisch Unterbeschäftigten auf dem Lande wirken, der darüber hinaus dann auch hier die Einkommenssituation (der verbleibenden Bauernhaushalte) verbessern dürfte. Die damit auch höher werdenden Verbrauchsausgaben werden sich aller Erfahrung nach in einer überproportionalen Steigerung des Nahrungsmittelkonsums und möglicherweise auch des Verzehrs qualitativ höherwertiger Nahrungsmittel niederschlagen. Die Asian Development Bank erklärt so z. B. die steigenden Reis- und Weizenimporte damit, daß man bisher direkt konsumiertes Barley und Sorghum nun als Viehfutter verwenden würde ${ }^{12}$.

Die Autoren des hier besprochenen Sammelbandes suchen hingegen die zunehmende Verarmung der koreanischen Bauern zu belegen. Schauen wir uns einige der von ihnen vorgetragenen Argumente etwas näher an.

So führte nach Du-Yul Song die Einfuhr des Überschußgetreides aus den USA zu einem Preisverfall der inländischen Agrarprodukte. Der Getreidepreisindex sei so von 1956 $(=100)$ bis 1959 auf 73 gefallen (S. 26). Er sagt nicht, daß er anschließend kontinuierlich ge-

10 Lediglich Ilse Lenz, dies sei anerkennend erwähnt, bemerkt wenigstens in einer Fußnote (S. 161), daß sie keinen Vergleich in bezug auf Nordkorea oder anderer sozialistischerStaaten intendiere, da dieser wegen Mangels an Materialien über den realen Alltag der, industriellen Beziehungen" dort auch nicht möglich sei. Ubrigens, warum wohl nicht?

11 Vgl. dazu aber: Asian Development Bank: Asian Agricultural Survey 1976, Manila 1977, p. 67, 98.

12 A. a. O., p. $39,41$. 
stiegen ist: Der Index fiel zwar in der Tat bis 1958 auf 73 (1959 ist bei Do-Yul Song offenbar ein Abschreibfehler), kletterte 1959 aber wieder auf 86, 1960 auf 106 usw. ${ }^{13}$.

Sowohl Du-Yul Song (S. 27) als auch Hans Luther (S. 93) berichten über die niedrigen staatlichen Aufkaufpreise für Reis. Diese sollen 1976 sogar um $15 \%$ unter den Produktionskosten gelegen haben (sie berufen sich dabei auf Angaben eines Katholischen Bauernverbandes). Damit unterstellen sie, daß die Bauern durch den Staat zum Desinvestieren (und letztlich zum Verkauf ihrer Parzellen) gezwungen werden. Sie verschweigen aber, daß der Staat zu seinen Preisen nur einen Teil des Getreides aufkauft (der zwar ständig erhöht wurde und 1975 ca. die Hälfte des Marktanteiles betrug), der freie Marktpreis immer erheblich über den Produktionskosten und eben auch über den staatlichen Aufkaufpreisen lag.

Schließlich lagen die staatlichen Aufkauf preise zwar von 1948 bis 60 zum Teilganz erheblich unter den Produktionskosten, seitdem (mindestens bis 1975) aber immer darüber. Dies kann man aus amtlichen Statistiken entnehmen, die dankenswerterweise Eddy Lee ${ }^{\mathbf{1 4}}$ zusammengestellt hat ${ }^{15}$.

Damit soll allerdings nicht bestritten werden, daß der Staat sich um eine Senkung der Grundnahrungsmittel- und Produzentenpreise bemüht hat und auch sonst der Agrarsektor zur Subventionierung der Industrie immer wieder herhalten mußte ${ }^{\mathbf{1 6}}$.

Jedenfalls hat aber kontinuierlich auch kein ,, ungleicher Tausch" im Sinne der Verschlechterung der terms-of-trade der Agrarprodukte zu den nicht-landwirtschaftlichen Gütern stattgefunden, wie sie Du-Yul Song behauptet und nur für 1965 bis 68 (richtig) belegt (S. 27); 1969 bis 1973 sind sie jedenfalls gestiegen ${ }^{17}$.

Wie hier die Zahlen manipuliert werden, mag noch folgendes Zitat verdeutlichen: , Ein weiterer Mechanismus, der zur andauernden Misere der südkoreanischen Bauern beigetragen hat, ist die rapide Kostensteigerung für Kunstdünger, Saatgut und Landmaschinen. Allein im Jahre 1976 stieg der Preis für Kunstdünger um 79,2 \%.“"(Luther, S. 93). Der Autor verschweigt, daß in den Jahren zuvor die Regierung sich um eine Stabilisierung der Düngemittelpreise wie auch der Produzentenpreise bemüht hatte. Dabei wurden allerdings so hohe Defizite akkumuliert, daß man zu den Marktpreisen zurückkehren mußte, was eben zu besagt enormen Preissteigerungen führte. Diese waren sicherlich politisch unklug, aber sind eben aus der vorliegenden Subventionsperiode zu erklären und zu verstehen ${ }^{\mathbf{1 8}}$.

Auch in Südkorea ist es sicherlich zu einer weiteren sozialen Differenzierung der Landbevölkerung gekommen, hat $u$. a. auch der Anteil der Landarbeiter und Pächter unter den Landbewirtschaftern wieder zugenommen (allerdings nicht in einem so dramatischen Umfang wie in den meisten anderen asiatischen Ländern, vielleicht mit Ausnahme Taiwans) ${ }^{\mathbf{1 9}}$.

13 Eddy Lee: Egalitarian peasant farming and rural development. The case of South Korea, in: Ghai, D. et. alii. (eds.): Agrarian Systems and rural development, London 1979, p. 60.

14 A. a. O., p. 60.

15 Seither scheinen die staatlichen Aufkaufpreise aber wieder unter den Produktionskosten zu liegen, wenn man den Angaben des Katholischen Bauernverbandes folgt, die Hans Luther in einer neueren Fassung seines Aufsatzes für 1975-78 zitiert. (Hans-U. Luther: Seamaul Undong: The „modernization“ of rural poverty in South Korea, in: Internationales Asienforum, 3/4, November 1979, p. 350). Die von Luther zitierten Zahlen stimmen zwar in der Tendenz, nicht aber in der absoluten Höhe mit den Angaben der auf derselben Quelle basierenden Publikation der Commission of the Churches on International Affairs of the World Council of Curches, Human rights in the Republic of Korea, Geneva, 1979/1, p. 12, überein. Die neuere Entwicklung ist natürlich auch kein Beleg für die "Landflucht" der sechziger und frühen siebziger Jahre.

16 Beispiele: Düngungsmittelindustrie: Suck-Kyo Ahn, S. 123, Luther, S. 93; Zementindustrie: Luther, S. 90.

17 Hierbei unterscheidet sich Südkorea durchaus vorteilhaft von den meisten anderen asiatischen Ländern. Vgl. dazu: Asian Development Bank: Asian Agricultural Survey 1976, Manila 1977, p. 160.

18 Ho spricht davon, daß allein 1974 die Defizite des Fertilizer- wie des Grain Management Fund $2 \%$ des BSP ausgemacht hätten. Die Preiserhöhungen für Düngemittel gibt er 1975 mit $65 \%$ und 1976 mit $69 \%$ an. Samuel P. S. Ho: Rural-urban inbalance in South Korea in the 1970s. Asian Survey, vol. XIX, Nu 7, July 1979, p. 651.

19 So nahm der Anteil der Landarbeiter von 9,7\% (1966) auf 14,5 \% (1972) zu. (ADB, a. a. O., p. 56). Der Anstieg der Pachtfläche an der Gesamtfläche stieg nach amtlichen Angaben von $8 \%$ (1953) auf 13,5\% (1960), 17,6\% (1970), 14,6\% (1975); der Anteil der reinen Pächterhaushalte an allen landwirtschaftlichen Betrieben hingegen nur von $5 \%$ (1953) auf 9,7 \% (1970), 7,9 \% (1975). Unabhängige Beobachter schätzen die Pachtfläche auf 20 bis $25 \%$ der gesamten Betriebsfläche. Lee, a. a. O., p. 54; Luther, p. 91 , spricht ohne Quellenangabe bzw. Schätzgrundlage - von 30 bis $45 \%$ je Provinz. 
Andererseits sind offenbar die Landarbeiterlöhne auch real tatsächlich gestiegen ${ }^{20}$, und über den Charakter dieser neuen Pachtverhältnisse wissen wir eigentlich nicht viel21.

Faßt man alle verfügbaren Daten zusammen, so spricht einiges dafür, daß auch die Realeinkommen der relativ ärmeren Bauernhaushalte - mindestens bis Mitte der siebziger Jahre gestiegen sind, vielleicht geringer, als bei einer anderen staatlichen Politik möglich gewesen wäre, und auch geringer als die der urbanen Haushalte.

Das absolute Niveau der durchschnittlichen ländlichen Einkommen ist sicher noch sehr niedrig. Dies trifft auch auf die städtischen Einkommen und insbesondere auf die Industriearbeiterlöhne zu. Ilse Lenz (S. 127, 131) spricht davon, daß diese in den letzten Jahren (nach 1970) leicht angestiegen seien. Dennoch sind diese nach wie vor erbärmlich niedrig. Liegen sie allerdings unter dem Existenzminimum? Reicht der Lohn nicht aus, daß die Arbeiter(innen) sich angemessen ernähren, kleiden, wohnen können, mit fatalen(langfristigen) Folgen für Gesundheit und Arbeitsfähigkeit? Ilse Lenz trägt hier einige Daten zusammen, die diese Annahme zu bestätigen scheinen (S. 127-129). Andererseits berichtet sie aber auch davon, daß viele Arbeiterinnen vom Lande in die Industrie gehen, um Geld für die Ausbildung ihrer kleineren Geschwister zu verdienen und z. T. bis zur Hälfte ihres Gehaltes nach Hause überweisen (bei gelegentlicher Mitnahme von Nahrungsmitteln) (S. 136/137). Trifft dies zu, würde das aber mehr dafür sprechen, daß die Lohneinkommen über dem Existenzminimum liegen bzw. durch Selbstbeschränkung Ersparnisse gebildet werden können. Dem widerspricht auch nicht die Tatsache, daß die südkoreanischen Arbeiter(innen) unzufrieden mit ihren Arbeitsbedingungen und Löhnen sind und in jüngster Zeit dies (durch Arbeitsniederlegungen usw.) auch zunehmend zeigen.

VIII.

Allerdings haben die beiden Olkrisen und Olpreisverteuerungen (1973 und 1979) Südkorea und das südkoreanische Modell zweifellos hart getroffen, härter getroffen, als andere Länder, die nicht so extrem auf den Außenwirtschaftssektor in ihrer Wachstums- und Entwicklungsstrategie gesetzt haben. Damit rutschte die Zahlungsbilanz in immer größere Defizite, wurde die Inflation immer mehr angeheizt, die nun, trotz nominaler Lohnerhöhungen von um die $30 \%$ in den letzten Jahren, auch die Reallöhne stagnieren oder gar sinken ließen: Die Konsequenz wurde schon erwähnt: die zunehmende soziale Mobilisierung der Arbeiterschaft. Damit stiegen aber auch die Lohnstückkosten schneller als in konkurrierenden anderen asiatischen Ländern. Hinzu gesellten sich allgemeine Absatzschwierigkeiten sowohl für die Industriewarenexporte aufgrund allgemeiner rezessiver Tendenzen auf dem Weltmarkt ${ }^{22}$ als auch der Bauwirtschaft im Nahen Osten aufgrund der Umschichtung der dortigen Entwicklungsvorhaben (weg von einfachen Infrastrukturbauvorhaben, hin zum Bau ganzer Industriekomplexe - für die die Koreaner bisher noch nicht das Know-how haben).

Derartige wirtschaftliche Streßsituationen sind die koreanischen Unternehmen - aufgrund

20 Dazu tendenziell gleichlautende, aber absolut, z. T. erheblich differierende Angaben bei der ADB, a. a. O., p. 54 und bei Lee, a. a. O., p. 33

21 Es wäre hier zu fragen, ob durch die Abwanderung u. U. ganzer Bauernhaushalte mit (bisher) Minibetrieben nicht andere Kleinbetriebe durch Zukauf oder auch Pacht sich nicht (etwas) arrondieren konnten oder ob ein neuer ausbeuterischer Landlordismus sich hier auszubreiten beginnt. Luther, S. 91, unterstellt das Letztere - mit Hinweis auf einen Zeitungsbericht. Sollte das Eindringen des Kapitalismus in die Landwirtschaft und die Landspekulation urbaner Mittelklasseelemente tatsächlich nicht nur Einzelerscheinungen sein, würde dies eigentlich auch der gesamten Argumentation des hier rezensierten Buches widersprechen.

22 Einige neuere Daten (bis 1979/80) sind zu finden in dem sonst auch analytisch interessanten Artikel von Ron Richardson, ,,South Korea/Taiwan: The battle to span the energy gap“. Far Eastern Economic Review, August 8-14, 1980, p. 40-45. 
ihrer Unterkapitalisierung - weit weniger zu meistern in der Lage, als dies möglicherweise sonst der Fall sein dürfte. Ausländische Investoren dürften zudem unter diesen Bedingungen bald keinen Sinn mehr sehen, in diesem Land zu investieren und zu produzieren ${ }^{23}$.

Diese Außenwirtschaftskrise, verbunden mit der dadurch immer brennender werdenden sozialen und politischen Krise, die Mobilisierung der Arbeiterschaft und der kleinbürgerlichen Opposition wie auch die Machtkämpfe innerhalb der führenden Militär- und Bürokratenclique, drohen das ganze System nun wie ein Kartenhaus zusammenfallen zu lassen. Ob dieser Zusammenbruch tatsächlich abgewendet werden kann und damit unter Beweis gestellt wird, daß Südkorea eine ernst zu nehmende Entwicklungsstrategie begonnen hat und diese - auf einer höheren Ebene - fortzuführen in der Lage ist, wird im wesentlichen nun von der Lösung von zwei Problemkomplexen abhängen:

Notwendig ist zunächst einmal die Úberwindung der sozialen und politischen Krise. Das kann nur gelingen, wenn die Arbeiter stärker am Wirtschaftssystem partizipieren, was steigende Reallöhne impliziert.

Es bedarf ferner in Zukunft einer institutionalisierten kritischen Úberprüfung der Prioritäten der Politik wie auch der Implementierungsmängel, dies impliziert eine demokratische Offnung des Regimes. Zu beidem sind die Machteliten (noch) nicht bereit. Sie glauben offenbar, daß ihre weitere diktatorische Machterhaltung mit den Mitteln des Terrors von oben hier noch genügt - wie es ja auch das Park-Regime nach 1961 lange Zeit eigentlich recht effizient vermocht hatte.

Nur ist die koreanische Gesellschaft von heute eine andere als die vor 20 Jahren. Die Proletarisierung vormals z. T. subsistent wirtschaftender kleiner (agrarischer) Warenproduzenten, verbunden mit der Ausbildungsrevolution und der Siedlung und Arbeit in großen urbanen Agglomerationen mit ihrer hohen Kommunikationsdichte, haben die Abhängigkeit von den gesellschaftlichen Produktionsverhältnissen und Bedingungen erhöht, das Bewußtsein darüber geschärft, den Bedürfnishorizont erweitert, schließlich die Bereitschaft und die Möglichkeit zu kollektiven sozialen und politischen Aktionen verbessert. Ein repressives, hochgerüstetes, von nicht allzu vielen moralischen Skrupeln eingeschränktes Regime, das sich wenigstens auf(kleine) Teile der Bevölkerung stützen kann, mag sich dennoch (vorläufig) an der Macht halten können (obwohl man da, nach den erfolgreichen Volksaufständen gerade in der letzten Zeit, auch nicht mehr so sicher sein sollte). Ob es dabei die „Grabesruhe“ an der Basis wiederherzustellen und zu garantieren vermag, muß stark bezweifelt werden. Das koreanische Wirtschaftsmodell mit sich zunehmend intensivierenden Konflikten in den Betrieben, mit Arbeitsniederlegungen, Streiks, Demonstrationen, Sit-ins, stillem Klassenkampf (passiver Sabotage, Arbeitsverschleppungen usw.), ist kaum vorstellbar.

Darüber hinaus hängt die wirtschaftliche Stabilität und weitere Entwicklung Koreas - gerade in der gegenwärtigen Phase - entscheidend auch davon ab, ob es gelingt, die Außenwirtschaftsstrategie auf andere als die komparativen Vorteile in den Lohnkosten und auf Staatssubventionen zu stützen und in Bereiche vorzudringen, in denen es zunehmend mehr auf die Beherrschung, Entwicklung und das Angebot komplizierter Technologien bzw. auf den Einsatz technisch qualifizierter (und damit nicht nur noch ,,billigerer") Arbeitskräfte ankommt. Nach allem, was wir wissen, scheinen die Vorarbeiten und Aussichten hier gleichfalls nicht besonders günstig zu sein. Andererseits war Südkorea auf wirtschaftlichem Gebiet bisher allemal für eine Uberraschung gut.

23 Der Journalist Andreas Kohlschütter berichtet in „,DIEZEIT“ (Südkorea: Ende einer Illusion, 15. 8. 1980, S. 10) in einem auch sonst informativen Artikel, daß 500 von 1300 joint ventures zwischen koreanischen und ausländischen Partnern von den ausländischen Unternehmern inzwischen nicht mehr erneuert worden seien. 